OPEN ACCESS

Edited by:

Manuela Uda,

National Research Council (CNR), Italy

Reviewed by:

Marjut Susanna Otala,

Helsinki University Central Hospital,

Finland

Jürgen Michael Weiss,

University of Lucerne, Switzerland

*Correspondence:

Zhengchao Wang

zcwang@fjnu.edu.cn

${ }^{\dagger}$ These authors have contributed equally to this work

Specialty section:

This article was submitted to

Reproduction,

a section of the journa

Frontiers in Physiology

Received: 15 August 2020 Accepted: 09 October 2020

Published: 05 November 2020

Citation:

Zhang $\mathrm{H}$, Lin F, Zhao J and Wang Z (2020) Expression Regulation and Physiological Role of Transcription Factor FOXO3a

During Ovarian Follicular

Development.

Front. Physiol. 11:595086.

doi: 10.3389/fphys.2020.595086

\section{Expression Regulation and Physiological Role of Transcription Factor FOXO3a During Ovarian Follicular Development}

\author{
Hong Zhang ${ }^{1+}$, Fengping Lin ${ }^{1+}$, Jiuhua Zhao ${ }^{1,2+}$ and Zhengchao Wang ${ }^{1 *}$ \\ ${ }_{1}^{1}$ Provincial Key Laboratory for Developmental Biology and Neurosciences, Provincial University Key Laboratory of Sport \\ and Health Science, Key Laboratory of Optoelectronic Science and Technology for Medicine of Ministry of Education, \\ College of Life Sciences, Fujian Normal University, Fuzhou, China, ${ }^{2}$ West Anhui Health Vocational College, Lu'an, China
}

In mammals, developing ovarian follicles transform from primordial follicles to primary follicles, secondary follicles, and mature follicles, accompanied by changes in follicular secretory functions. FoxO3a is a member of the forkhead transcription factor family (FoxO), which plays an important role in the cell cycle, DNA damage repair, apoptosis, oxidative stress, and energy metabolism. Recent studies have shown that $\mathrm{FOXO} 3 \mathrm{a}$ is involved in the physiological regulation of follicular development and pathological progression of related ovarian diseases, which will provide useful concepts and strategies for retarding ovarian aging, prolonging the ovarian life span, and treating ovarian diseases. Therefore, the regulation of FOXO3a expression, as well as the physiological contribution during ovarian follicular development are detailed in this paper, presenting an important reference for the further study of ovarian biology.

Keywords: transcription factor FOXO3a, cell cycle, apoptosis, oxidative stress, energy metabolism, follicular development

\section{INTRODUCTION}

Follicular development is a complex reproduction-related physiological process characterized by cell proliferation, differentiation, and apoptosis. Typically, based on morphology and function, follicular development can be artificially divided into different stages, including primordial follicles, primary follicles, secondary follicles, and mature follicles (Wei et al., 2012, 2019; Huang et al., 2016; Wu et al., 2019). Notably, various diseases could be induced by follicular dysplasia, including premature ovarian failure, polycystic follicular syndrome, and infertility (Yang et al., 2010; Thanatsis et al., 2019). Forkhead box (Fox) proteins are highly conserved transcription factors structurally, currently attracting a great deal of attention. Among them, FOXO3a is an important member, and its discovery originates from its homologous protein DAF-16, which is also a well-studied transcription factor (Liu et al., 2018). Ogg et al. (1997) revealed that the FOXO3a homologous protein, DAF-16, is negatively regulated by the insulin signaling pathway. Furthermore, it participates in the regulation of the cell cycle and life expectancy, which is closely related to the lifespan, metabolism, and reproduction of worms (Ogg et al., 1997). Thus, FOXO3a may be closely related to the development, metabolism, and other functions of organisms. Recent studies have shown that FOXO3a is involved in follicular development, thus presenting a valuable 
target for the study of follicular development, and displays important theoretical and practical significance for better understanding the mammalian reproductive mechanism.

\section{THE DEVELOPMENT OF OVARIAN FOLLICLES}

In mammals, the primordial follicle is the basic female reproductive unit and the only form of ovarian cell reserve (Wei et al., 2012, 2019; Huang et al., 2016; Wu et al., 2019). Primitive follicular pools are formed during early life such as the late embryonic stage in humans and the fourth day after birth in rats (Tang et al., 2017). Furthermore, once primordial follicles are formed, their total number remains fixed and is no longer increased. Usually, after the formation of primordial follicles, there will be a continuous batch of developing primordial follicles, and then forming follicles at different developmental stages, finally becoming dominant follicles triggering ovulation to commence a new life journey (Zhang Z. et al., 2019). During follicular development, most follicles die a programmed death or degeneration, which is termed follicular apoptosis or atresia (Tang et al., 2017). There are two types of follicular atresia from the morphological standpoint, starting from oocytes or granulosa cells, respectively (Manabe et al., 2004; Shimizu et al., 2009).

\section{TRANSCRIPTION FACTOR FOXO3a}

The first forkhead protein was discovered in Drosophila melanogaster by Weigel et al. (1989). To date, more than 100 family members have been verified, from FOXA to FOXS (Anderson et al., 1998; Lee and Dong, 2017; Murtaza et al., 2017). FOXO belongs to the "O" class of the FOX superfamily. In mammals, this group contains four members: FOXO1/FKHR/FOXO1a, FOXO3/FKHRL1/FOXO3a, FOXO4/AFX, and FOXO6 (Murtaza et al., 2017). All FoxO proteins share a highly conserved DNA-binding domain, presenting 110 amino acids folded into three $\alpha$-helixes and two wing-like large loops. In addition, the structure includes a nuclear localization signal, a nuclear export signal motif, and a C-terminal transcriptional active region (Obsil and Obsilova, 2008). These proteins are ubiquitously expressed in various tissues throughout the body, except for FOXO6, which currently has been reported only in the adult brain tissue (Jacobs et al., 2003). Notably, the Human Protein Atlas ${ }^{1}$ indicates that the expression of FOXO3 in human ovarian stromal and follicular cells is abundant.

\section{EXPRESSION REGULATION OF FOXO3a DURING FOLLICULAR DEVELOPMENT}

During recent years, several studies have investigated the regulation of FOXO3a expression. The activity of FOXO3a

${ }^{1}$ http://www.proteinatlas.org can be improved at multiple levels, in which post-translational modification is the main approach, including phosphorylation, acetylation, and ubiquitination (Figure 1).

\section{Phosphorylation and Dephosphorylation}

FOXO3a can be phosphorylated by kinases such as protein kinase $B(\mathrm{PKB})$, extracellular-regulated kinase, serum and glucocorticoid-induced kinase, and inhibitor kappa $B$ kinase $\beta$ (Brunet et al., 2001). The transcriptional regulation of FOXO3a is closely related to phosphoinositide-3 kinase (PI-3K)/protein kinase $\mathrm{B}(\mathrm{PKB})$ signaling, which was first proposed by Brunet et al. (1999). In mammals, FOXO3a can be phosphorylated by PKB in the nucleus and then transported from the nucleus to the cytoplasm, utilizing the 14-3-3 molecular chaperone after the activation of insulin signaling. FOXO3a is translocated into the cytoplasm and could bind with the polyubiquitination system, to be subsequently degraded by proteasomes (Plas and Thompson, 2003; Wang et al., 2015), which accompanies the transcriptional activity loss of FOXO3a, which no longer plays a regulatory role during cell development and metabolism (Datta et al., 1999; Brunet et al., 2002; Plas and Thompson, 2003, 2005). Therefore, the localization of FOXO3a in the cytoplasm not only inactivates its function but is also extremely crucial for the degradation of FOXO3a.

Phosphatase and tensin homolog deleted on chromosome ten (PTEN) is a key negative regulator for PI3K/PKB signaling, which can improve the suppression of FOXO3a through dephosphorylation (Ding et al., 2010; Jang et al., 2016; Li J. et al., 2020). Additionally, endogenous PKB and FOXO3a can form a complex. Furthermore, FOXO3a can negatively regulate PKB and its downstream molecules (Takaishi et al., 1999; Lin et al., 2001; Junger et al., 2003; Puig et al., 2003). Simultaneously, elevated 14-3-3 can increase FOXO3a expression and phosphorylation, maintaining the phosphorylated FOXO3a protein stability (Cahill et al., 2001).

Reddy et al. (2005) reported the activation of PKB and suppression of FOXO3a in mouse and rat oocytes using stem cell factor (SCF) during follicular development. Meng et al. (2007) showed the stage/cell-specific expression patterns of FOXO3a and $\mathrm{PKB}$, suggesting that these proteins might play potential roles in the follicular development of the mini-pig. Furthermore, consistent results were observed in fetal and neonatal pig ovaries (Ding et al., 2010). These results indicate the important role of $\mathrm{PKB} / \mathrm{FOXO} 3 \mathrm{a}$ and the impact of PKB regulation on FOXO3a phosphorylation during follicular development.

\section{Acetylation and Deacetylation}

In addition to the regulation of PKB, FOXO3a is also mediated via acetylation and deacetylation (Kim et al., 2010; Xiang et al., 2012; Zhang et al., 2013; Wang et al., 2014; Zhou et al., 2014; Liu et al., 2015; Long et al., 2019). CREB binding protein, p300, and other nuclear proteins can acetylate lysine on the DNA-binding region of FOXO3a protein, resulting in reduced FOXO3a transcriptional activation (Watroba et al., 2012). Sirtuin 1 (SIRT1) is a NAD-dependent histone deacetylase (Long et al., 2019). Typically, SIRT1 in the nucleus may activate the transcriptional activity of FOXO3a, regulating cellular functions 


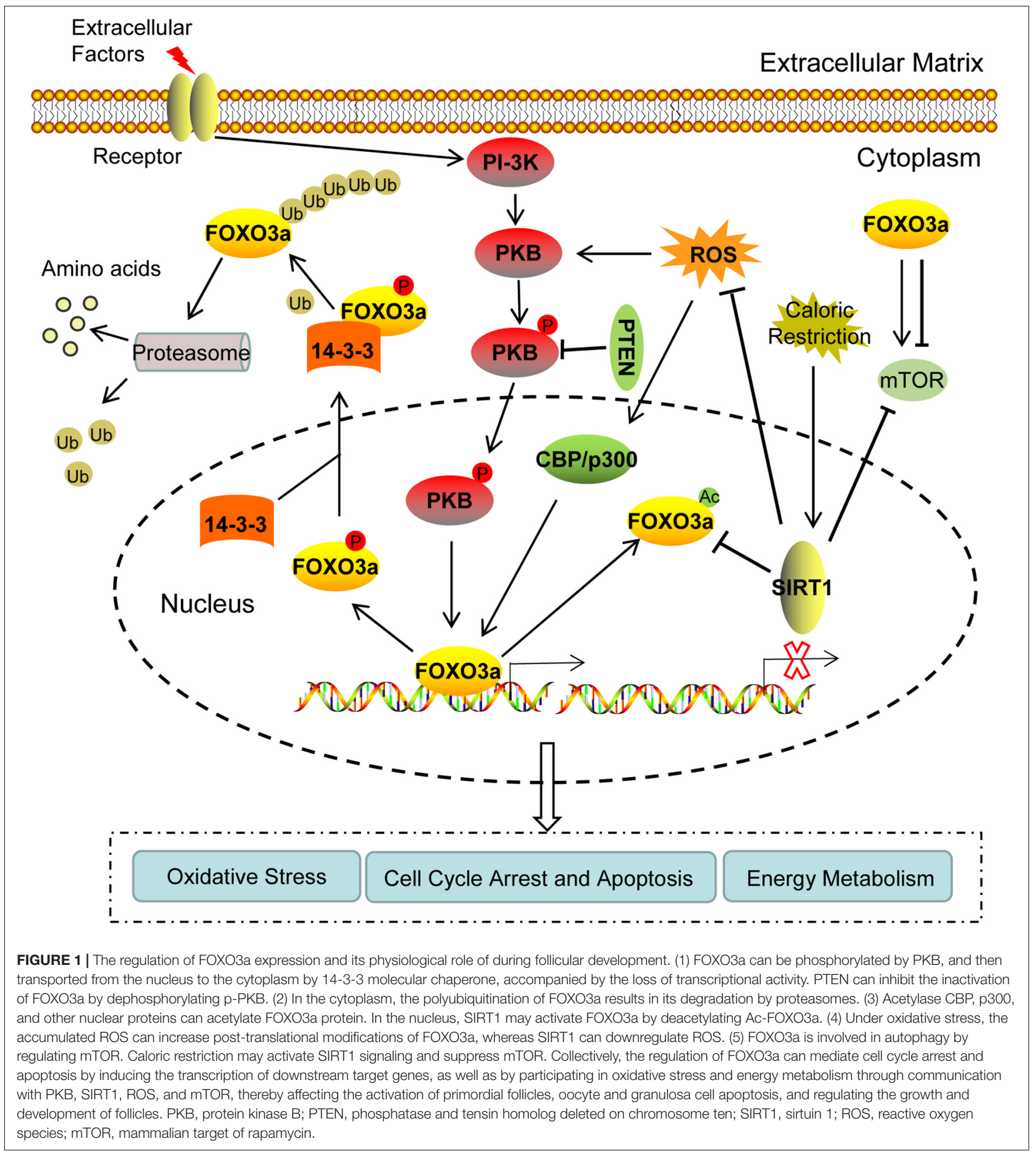

by deacetylating Ac-FOXO3a (Glauser and Schlegel, 2007). Gorczyca et al. (2019) demonstrated the presence of SIRT1 and SIRT6 in ovarian cells, and their involvement in the control of follicular atresia. Recent studies revealed that energy restriction can increase the expression of SIRT1 and activate SIRT1related signaling pathways in adult mice (Xiang et al., 2012;
Zhang et al., 2013; Liu et al., 2015; Long et al., 2019). Additionally, Kim et al. (2010) reported that the high expression of FOXO3a can upregulate SITR6 activity, whereas the inhibition of FOXO3a expression could downregulate SIRT6 activity. Simultaneously, the downregulation of FOXO3a can prevent the effect of SIRT6 on energy limitation, as well as on SIRT1 (Kim et al., 2010). 


\section{PHYSIOLOGICAL ROLES OF FOXO3a DURING FOLLICULAR DEVELOPMENT}

Currently, numerous studies have presented that FOXO3a is associated with follicular development (Brenkman and Burgering, 2003; Adhikari and Liu, 2009; Monniaux et al., 2016). Experimental studies have reported that female FOXO3a knockout mice exhibit global follicular activation at an early stage of follicular growth, leading to oocyte death, early depletion of functional ovarian follicles, and secondary infertility (Castrillon et al., 2003). Conversely, FOXO3 overexpression can delay the development of primordial follicles, increase the follicular reserve, and ovarian reproductive capacity in mice. Compared with wild-type littermates, increased follicle numbers and decreased gonadotropin levels were documented in aging FOXO3-transgenic mice (Pelosi et al., 2013). Thus, FOXO3a may play an important role in maintaining the pool number of primordial follicles and the physiological functions of the ovarian reserve, as well as female fertility. Furthermore, some researchers have reported that the FOXO3 protein regulates follicle growth and atresia by promoting apoptosis of granulosa cells and oocytes in mammalian ovaries (Liu et al., 2009; Matsuda et al., 2011).

Although the function of FOXO3a in ovarian follicle development has been relatively known, its mechanism remains unclear. It is generally accepted that FOXO3a is widely involved in the cell cycle, DNA damage repair, apoptosis, oxidative stress, and metabolism. Hence, we presented evidence postulating that the role of FOXO3a in follicular development is related to these processes (Figure 1).

\section{Cell Cycle Arrest and Apoptosis}

FOXO3a activity impacts the expression of downstream target genes, resulting in cell cycle and apoptotic disturbances (Medema et al., 2000). FoxO3a can increase the expression of the cyclin-dependent kinase inhibitor protein, p27kip, and decrease the expression of cyclin $\mathrm{D}$ in the nucleus, maintaining cells in a stationary phase and inhibiting follicular development (Schmidt et al., 2002). Liu et al. (2009) suggested that FOXO3a is involved in oocyte apoptosis in the neonatal rat ovary, and the SCF-PI3K/PKB-FOXO3a signaling pathway mediates primordial follicle formation and oocyte apoptosis by regulating the expression of p27kip1 and proapoptotic factors such as Bim, Bad, and Bax. Moreover, research on chicken primary ovarian granulosa cells indicated that in the absence of FOXO3, mRNA levels of proapoptotic factors BNIP3 and BCL2L11 decreased, along with poly [ADP-ribose] polymerase 1 (PARP1) and cleaved caspase 3 protein levels. After treatment with a recombinant FOXO3 protein, mRNA levels of BNIP3 and BCL2L11, as well as protein levels of PARP-1 and caspase3, were reportedly increased (Cui et al., 2019). Experiments in human ovarian granulosa-like tumor cells (KGN) have shown that expression of the proapoptotic factors FASLG and BCL2L11 is upregulated and cell death is induced by transfection of FOXO3 expression vectors (Matsuda et al., 2011). Collectively, these studies have consistently demonstrated that FOXO3 is expressed in reproductive tissues, including ovarian oocytes and granulosa cells, and promotes apoptosis.

\section{Oxidative Stress}

Reportedly, accumulated evidence suggests that oxidative stress is associated with disrupted follicular development, which may result in increased follicular atresia (Yan et al., 2020). Under oxidative stress, accumulated reactive oxygen species (ROS) leads to post-translational modifications of FOXO3a, thereby regulating the activity and function of FOXO3a. Park et al. (2020) demonstrated that SIRT1 can downregulate ROS and form a complex with FOXO3a in cells, which can improve the ability of FOXO3a to induce cell cycle arrest and promote cell survival. Recent findings have indicated that resveratrol, a plant polyphenolic compound, can enhance SIRT1 and decrease ovarian oxidative stress as well as inhibit phosphorylation of p66Shc, both in vivo and in vitro (Wang et al., 2020). Thus, in terms of follicular development, there undoubtedly exists an interactive relationship between ROS, SIRT1, and FOXO3a. However, the specific mechanism needs to be elucidated.

\section{Energy Metabolism}

Mammalian target of rapamycin (mTOR) is a major negative regulatory factor of autophagy (Choi et al., 2011). It has been previously reported that $\mathrm{PKB}$-mediated activation of mTOR inhibits granulosa cell autophagy during follicular development (Choi et al., 2014). Growing evidence strongly indicates that FOXO3a is involved in autophagy. If abundant energy is available, the modification of FoxO3 inhibits its activity, thereby decreasing the transcription of autophagy genes and downregulating autophagy. However, PI3K-PKB-FOXO3 can promote autophagy by mediating mTOR inhibition (van der Vos et al., 2012). Long et al. (2019) reported that oocytespecific SIRT1-overexpressing mice demonstrated an improved follicle reserve and a prolonged ovarian lifespan by continuously activating FOXO3a and suppressing mTOR. Furthermore, SIRT1 can facilitate primordial follicle recruitment through directly modulating $\mathrm{PKB}$ and $\mathrm{mTOR}$ transcription, independent of deacetylase activity (Zhang T. et al., 2019). High-fat diet-induced obesity may accelerate ovarian follicle development and the rate of follicle loss by activating mTOR and suppressing SIRT1 signaling. Caloric restriction may improve the adverse effects of high-fat diet-induced obesity on ovarian follicles (Xiang et al., 2012; Wang et al., 2014; Li et al., 2015; Liu et al., 2015). Thus, FOXO3a, mTOR, PKB, and SIRT1 may be implicated in autophagy and energy metabolism during follicular development.

\section{FOXO3a AND OVARIAN DISEASE}

Reportedly, the deletion of FOXO3a, FOXL2, PTEN, and p27 leads to early exhaustion of the primordial follicle pool and premature ovarian insufficiency in transgenic mice (Thanatsis et al., 2019). Melatonin prevents cisplatin-induced primordial follicle loss by suppressing the PTEN/AKT/FOXO3a pathway in the mouse ovary (Jang et al., 2016). Li Y. et al. (2020) observed that 
oral oyster polypeptide can protect the ovaries from $\mathrm{D}$-galactoseinduced premature ovarian failure, mediated via anti-oxidative stress activity. Meanwhile, growing data demonstrate that excess androgen may be the primary cause of polycystic ovary syndrome (PCOS). During the early stage of mouse folliculogenesis, testosterone induces the redistribution of FOXO3a, suggesting the involvement of FOXO3a in the pathogenesis of PCOS (Yang et al., 2010).

It has been well established that ovarian cancer presents the highest mortality rate among gynecological malignancies. Reportedly, FOXO3a expression can be increased by LSD1 knockdown, thereby inhibiting the proliferation and metastasis of ovarian cancer HO8910 cells (Liu et al., 2020). Kaplan-Meier survival analysis suggested that the low expression of FOXO3a was significantly related to poor prognosis in ovarian cancer patients (Fei et al., 2009). Recently, Xia et al. (2020) revealed that microRNA-506-3p inhibits proliferation and promotes apoptosis in ovarian cancer cells by targeting the AKT/FOXO3a signaling pathway. O'Neill et al. (2013) suggested that blocking the epidermal growth factor receptor (EGFR) results in PI3K-PKB inhibition and increases FOXO3a activation, which provides a new and valuable treatment strategy for breast cancer, prostate cancer, and ovarian cancer.

\section{SUMMARY AND CONCLUSION}

Based on the studies investigating the regulation of FOXO3a expression, it is currently established that FOXO3a can enhance the transcriptional regulation of its target genes, thereby enhancing its physiological contribution during the cell cycle and apoptosis regulation, resistance to oxidative stress, and prolongation of life span in organisms (Figure 1). Furthermore, FOXO3a signaling can induce oocyte and granulosa cell apoptosis, inhibit the activation of primordial follicles, and regulate the growth and development of follicles. The activation

\section{REFERENCES}

Adhikari, D., and Liu, K. (2009). Molecular mechanisms underlying the activation of mammalian primordial follicles. Endocr. Rev. 30, 438-464. doi: 10.1210/er. 2008-0048

Anderson, M. J., Viars, C. S., Czekay, S., Cavenee, W. K., and Arden, K. C. (1998). Cloning and characterization of three human forkhead genes that comprise an FKHR-like gene subfamily. Genomics 47, 187-199. doi: 10.1006/geno.1997. 5122

Brenkman, A. B., and Burgering, B. M. (2003). FoxO3a eggs on fertility and aging. Trends Mol. Med. 9, 464-467. doi: 10.1016/j.molmed.2003.09.003

Brunet, A., Bonni, A., Zigmond, M. J., Lin, M. Z., Juo, P., Hu, L. S., et al. (1999). Akt promotes cell survival by phosphorylating and inhibiting a Forkhead transcription factor. Cell 96, 857-868. doi: 10.1016/S0092-8674(00) 80595-4

Brunet, A., Kanai, F., Stehn, J., Xu, J., Sarbassova, D., Frangioni, J. V., et al. (2002). 14-3-3 transits to the nucleus and participates in dynamic nucleocytoplasmic transport. J. Cell Biol. 156, 817-828. doi: 10.1083/jcb.200112059

Brunet, A., Park, J., Tran, H., Hu, L. S., Hemmings, B. A., and Greenberg, M. E. (2001). Protein kinase SGK mediates survival signals by phosphorylating the forkhead transcription factor FKHRL1 (FOXO3a). Mol. Cell. Biol. 21, 952-965. doi: 10.1128/MCB.21.3.952-965.2001 of FOXO3a signaling can inhibit the developmental initiation of primordial follicles, maintain the initial state of primordial follicles, reduce the number of primordial follicles transformed into mature follicles, thus preserving the follicular reserves, delaying the depletion of follicles, and delaying the aging of ovaries. It is important to further explore the mechanism concerning the regulation of FOXO3a expression on its target genes, the physiological contribution of FOXO3a during ovarian follicular development, and its future clinical applications, further advancing the field of reproductive biology.

\section{AUTHOR CONTRIBUTIONS}

$\mathrm{HZ}, \mathrm{FL}$, and JZ wrote the manuscript and ZW revised it. All authors read and approved the final version of the manuscript for publication.

\section{FUNDING}

This work was supported by the Fujian Provincial Natural Science Foundation (2017J01626 and 2018J01722), Fujian Province Science and Technology Project of the Education Department (JZ160426), Research Program of Excellent Young Talents in Colleges and Universities of Anhui Provincial Education Department (GXGNFX2019120), the Education Reform Project (Y201809 and I202003009), and Training Program of Innovation and Entrepreneurship for Undergraduates (CXXL2020291 and CXXL2020293) of the Fujian Normal University.

\section{ACKNOWLEDGMENTS}

We would like to thank Editage (www.editage.com) for English language editing.

Cahill, C. M., Tzivion, G., Nasrin, N., Ogg, S., Dore, J., Ruvkun, G., et al. (2001). Phosphatidylinositol 3-kinase signaling inhibits DAF-16 DNA binding and function via 14-3-3-dependent and 14-3-3-independent pathways. J. Biol. Chem. 276, 13402-13410. doi: 10.1074/jbc.M010042200

Castrillon, D. H., Miao, L., Kollipara, R., Horner, J. W., and DePinho, R. A. (2003). Suppression of ovarian follicle activation in mice by the transcription factor Foxo3a. Science 301, 215-218. doi: 10.1126/science.1086336

Choi, J., Jo, M., Lee, E., and Choi, D. (2011). Induction of apoptotic cell death via accumulation of autophagosomes in rat granulosa cells. Fertil. Steril. 95, 1482-1486. doi: 10.1016/j.fertnstert.2010.06.006

Choi, J., Jo, M., Lee, E., and Choi, D. (2014). AKT is involved in granulosa cell autophagy regulation via mTOR signaling during rat follicular development and atresia. Reproduction 147, 73-80. doi: 10.1530/REP-13-0386

Cui, C., Han, S., Yin, H., Luo, B., Shen, X., Yang, F., et al. (2019). FOXO3 Is Expressed in Ovarian Tissues and Acts as an Apoptosis Initiator in Granulosa Cells of Chickens. Biomed. Res. Int. 2019:6902906. doi: 10.1155/2019/6902906

Datta, S. R., Brunet, A., and Greenberg, M. E. (1999). Cellular survival: a play in three Akts. Genes Dev. 13, 2905-2927. doi: 10.1101/gad.13.22.2905

Ding, W., Wang, W., Zhou, B., Zhang, W., Huang, P., Shi, F., et al. (2010). Formation of primordial follicles and immunolocalization of PTEN, PKB and FOXO3A proteins in the ovaries of fetal and neonatal pigs. J. Reprod. Dev. 56, 162-168. doi: 10.1262/jrd.09-094H 
Fei, M., Zhao, Y., Wang, Y., Lu, M., Cheng, C., Huang, X., et al. (2009). Low expression of Foxo3a is associated with poor prognosis in ovarian cancer patients. Cancer Invest. 27, 52-59. doi: 10.1080/07357900802146204

Glauser, D. A., and Schlegel, W. (2007). The emerging role of FOXO transcription factors in pancreatic beta cells. J. Endocrinol. 193, 195-207. doi: 10.1677/JOE06-0191

Gorczyca, G., Wartalski, K., Tabarowski, Z., and Duda, M. (2019). Effects of vinclozolin exposure on the expression and activity of SIRT1 and SIRT6 in the porcine ovary. J. Physiol. Pharmacol. 70, 153-165. doi: 10.26402/jpp.2019.1.15

Huang, P., Zhou, Z., Shi, F., Shao, G., Wang, R., Wang, J., et al. (2016). Effects of the IGF-1/PTEN/Akt/FoxO signaling pathway on male reproduction in rats subjected to water immersion and restraint stress. Mol. Med. Rep. 14, 5116-5124. doi: 10.3892/mmr.2016.5880

Jacobs, F. M., van der Heide, L. P., Wijchers, P. J., Burbach, J. P., Hoekman, M. F., and Smidt, M. P. (2003). FoxO6, a novel member of the FoxO class of transcription factors with distinct shuttling dynamics. J. Biol. Chem. 278, 35959-35967. doi: 10.1074/jbc.M302804200

Jang, H., Lee, O. H., Lee, Y., Yoon, H., Chang, E. M., Park, M., et al. (2016). Melatonin prevents cisplatin-induced primordial follicle loss via suppression of PTEN/AKT/FOXO3a pathway activation in the mouse ovary. J. Pineal. Res. 60, 336-347. doi: 10.1111/jpi.12316

Junger, M. A., Rintelen, F., Stocker, H., Wasserman, J. D., Vegh, M., Radimerski, T., et al. (2003). The Drosophila forkhead transcription factor FOXO mediates the reduction in cell number associated with reduced insulin signaling. J. Biol. 2:20. doi: 10.1186/1475-4924-2-20

Kim, H. S., Xiao, C., Wang, R. H., Lahusen, T., Xu, X., Vassilopoulos, A., et al. (2010). Hepatic-specific disruption of SIRT6 in mice results in fatty liver formation due to enhanced glycolysis and triglyceride synthesis. Cell Metab. 12, 224-236. doi: 10.1016/j.cmet.2010.06.009

Lee, S., and Dong, H. H. (2017). FoxO integration of insulin signaling with glucose and lipid metabolism. J. Endocrinol. 233, R67-R79. doi: 10.1530/JOE-17-0002

Li, J., Zhang, W., Zhu, S., and Shi, F. (2020). Nitric oxide synthase is involved in follicular development via the PI3K/AKT/FoxO3a pathway in neonatal and immature rats. Animals 10:248. doi: 10.3390/ani10020248

Li, L., Fu, Y. C., Xu, J. J., Lin, X. H., Chen, X. C., Zhang, X. M., et al. (2015). Caloric restriction promotes the reserve of follicle pool in adult female rats by inhibiting the activation of mammalian target of rapamycin signaling. Reprod. Sci. 22, 60-67. doi: 10.1177/1933719114542016

Li, Y., Qiu, W., Zhang, Z., Han, X., Bu, G., Meng, F., et al. (2020). Oral oyster polypeptides protect ovary against d-galactose-induced premature ovarian failure in C57BL/6 mice. J. Sci. Food Agric. 100, 92-101. doi: 10.1002/jsfa.9997

Lin, K., Hsin, H., Libina, N., and Kenyon, C. (2001). Regulation of the Caenorhabditis elegans longevity protein DAF-16 by insulin/IGF-1 and germline signaling. Nat. Genet. 28, 139-145. doi: 10.1038/88850

Liu, H., Luo, L. L., Qian, Y. S., Fu, Y. C., Sui, X. X., Geng, Y. J., et al. (2009). FOXO3a is involved in the apoptosis of naked oocytes and oocytes of primordial follicles from neonatal rat ovaries. Biochem. Biophys. Res. Commun. 381, 722-727. doi: 10.1016/j.bbrc.2009.02.138

Liu, L., Hao, F. X., Wang, A. P., Chen, X. L., Zhang, B., Wu, Z., et al. (2020). Lysine-specific demethylase 1 (LSD1) promotes ovarian cancer cell progression by Forkhead box O 3a (FOXO3a) inhibition. Mater. Express. 10, 594-602. doi: 10.1166/mex.2020.1674

Liu, W. J., Zhang, X. M., Wang, N., Zhou, X. L., Fu, Y. C., and Luo, L. L. (2015). Calorie restriction inhibits ovarian follicle development and follicle loss through activating SIRT1 signaling in mice. Eur. J. Med. Res. 20:22. doi: 10.1186/s40001-015-0114-8

Liu, Y., Ao, X., Ding, W., Ponnusamy, M., Wu, W., Hao, X., et al. (2018). Critical role of FOXO3a in carcinogenesis. Mol. Cancer 17:104. doi: 10.1186/s12943018-0856-3

Long, G. Y., Yang, J. Y., Xu, J. J., Ni, Y. H., Zhou, X. L., Ma, J. Y., et al. (2019). SIRT1 knock-in mice preserve ovarian reserve resembling caloric restriction. Gene 686, 194-202. doi: 10.1016/j.gene.2018.10.040

Manabe, N., Goto, Y., Matsuda-Minehata, F., Inoue, N., Maeda, A., Sakamaki, K., et al. (2004). Regulation mechanism of selective atresia in porcine follicles: regulation of granulosa cell apoptosis during atresia. J. Reprod. Dev. 50, $493-$ 514. doi: 10.1262/jrd.50.493

Matsuda, F., Inoue, N., Maeda, A., Cheng, Y., Sai, T., Gonda, H., et al. (2011). Expression and function of apoptosis initiator FOXO3 in granulosa cells during follicular atresia in pig ovaries. J. Reprod. Dev. 57, 151-158. doi: 10.1262/jrd.10$124 \mathrm{H}$

Medema, R. H., Kops, G. J., Bos, J. L., and Burgering, B. M. (2000). AFX-like Forkhead transcription factors mediate cell-cycle regulation by Ras and PKB through p27kip1. Nature 404, 782-787. doi: 10.1038/35008115

Meng, C., Shi, F., Zhou, Z., Huang, R., Liu, G., Watanabe, G., et al. (2007). Cellular Localization of Inhibin alpha-subunit, $\mathrm{PKB} / \mathrm{Akt}$ and FoxO3a proteins in the ovaries of minipigs. J. Reprod. Dev. 53, 229-236. doi: 10.1262/jrd. 18078

Monniaux, D., Michel, P., Postel, M., and Clement, F. (2016). Multi-scale modelling of ovarian follicular development: from follicular morphogenesis to selection for ovulation. Biol. Cell 108, 149-160. doi: 10.1111/boc.201500087

Murtaza, G., Khan, A. K., Rashid, R., Muneer, S., Hasan, S. M. F., and Chen, J. (2017). FOXO transcriptional factors and long-term living. Oxid. Med. Cell. Longev. 2017:3494289. doi: 10.1155/2017/3494289

Obsil, T., and Obsilova, V. (2008). Structure/function relationships underlying regulation of FOXO transcription factors. Oncogene 27, 2263-2275. doi: 10. 1038/onc.2008.20

Ogg, S., Paradis, S., Gottlieb, S., Patterson, G. I., Lee, L., Tissenbaum, H. A., et al. (1997). The Fork head transcription factor DAF-16 transduces insulinlike metabolic and longevity signals in C. elegans. Nature 389, 994-999. doi: 10.1038/40194

O’Neill, F., Madden, S. F., Clynes, M., Crown, J., Doolan, P., Aherne, S. T., et al. (2013). gene expression profile indicative of early stage HER2 targeted therapy response. Mol. Cancer 12:69. doi: 10.1186/1476-4598-12-69

Park, S., Shin, J., Bae, J., Han, D., Park, S. R., Shin, J., et al. (2020). SIRT1 alleviates LPS-induced IL-1beta production by suppressing NLRP3 inflammasome activation and ROS production in trophoblasts. Cells 9:728. doi: 10.3390/ cells 9030728

Pelosi, E., Omari, S., Michel, M., Ding, J., Amano, T., Forabosco, A., et al. (2013). Constitutively active Foxo3 in oocytes preserves ovarian reserve in mice. Nat. Commun. 4:1843. doi: 10.1038/ncomms2861

Plas, D. R., and Thompson, C. B. (2003). Akt activation promotes degradation of tuberin and FOXO3a via the proteasome. J. Biol. Chem. 278, 12361-12366. doi: 10.1074/jbc.M213069200

Plas, D. R., and Thompson, C. B. (2005). Akt-dependent transformation: there is more to growth than just surviving. Oncogene 24, 7435-7442. doi: 10.1038/sj. onc. 1209097

Puig, O., Marr, M. T., Ruhf, M. L., and Tjian, R. (2003). Control of cell number by Drosophila FOXO: downstream and feedback regulation of the insulin receptor pathway. Genes Dev. 17, 2006-2020. doi: 10.1101/gad.1098703

Reddy, P., Shen, L., Ren, C., Boman, K., Lundin, E., Ottander, U., et al. (2005). Activation of Akt (PKB) and suppression of FKHRL1 in mouse and rat oocytes by stem cell factor during follicular activation and development. Dev. Biol. 281, 160-170. doi: 10.1016/j.ydbio.2005.02.013

Schmidt, M., Fernandez de Mattos, S., van der Horst, A., Klompmaker, R., Kops, G. J., Lam, E. W., et al. (2002). Cell cycle inhibition by FoxO forkhead transcription factors involves downregulation of cyclin D. Mol. Cell. Biol. 22, 7842-7852. doi: 10.1128/MCB.22.22.7842-7852.2002

Shimizu, T., Kosaka, N., Murayama, C., Tetsuka, M., and Miyamoto, A. (2009). Apelin and APJ receptor expression in granulosa and theca cells during different stages of follicular development in the bovine ovary: involvement of apoptosis and hormonal regulation. Anim. Reprod. Sci. 116, 28-37. doi: 10.1016/ j.anireprosci.2009.01.009

Takaishi, H., Konishi, H., Matsuzaki, H., Ono, Y., Shirai, Y., Saito, N., et al. (1999). Regulation of nuclear translocation of forkhead transcription factor AFX by protein kinase B. Proc. Natl. Acad. Sci. U.S.A. 96, 11836-11841. doi: 10.1073/ pnas.96.21.11836

Tang, Z., Zhang, Z., Tang, Y., Qi, L., Yang, F., and Wang, Z. (2017). Effects of dimethyl carbonate-induced autophagic activation on follicular development in the mouse ovary. Exp. Ther. Med. 14, 5981-5989. doi: 10.3892/etm.2017. 5328

Thanatsis, N., Kaponis, A., Koika, V., Georgopoulos, N. A., and Decavalas, G. O. (2019). Reduced Foxo3a, FoxL2, and p27 mRNA expression in human ovarian tissue in premature ovarian insufficiency. Hormones (Athens) 18, 409-415. doi: 10.1007/s42000-019-00134-4

van der Vos, K. E., Eliasson, P., Proikas-Cezanne, T., Vervoort, S. J., van Boxtel, R., Putker, M., et al. (2012). Modulation of glutamine metabolism by the 
PI(3)K-PKB-FOXO network regulates autophagy. Nat. Cell Biol. 14, 829-837. doi: $10.1038 /$ ncb2536

Wang, D., Wang, T., Wang, R., Zhang, X., Wang, L., Xiang, Z., et al. (2020). Suppression of p66Shc prevents hyperandrogenism-induced ovarian oxidative stress and fibrosis. J. Transl. Med. 18:84. doi: 10.1186/s12967-020-02249-4

Wang, N., Luo, L. L., Xu, J. J., Xu, M. Y., Zhang, X. M., Zhou, X. L., et al. (2014). Obesity accelerates ovarian follicle development and follicle loss in rats. Metabolism 63, 94-103. doi: 10.1016/j.metabol.2013.09.001

Wang, Y. Q., Cao, Q., Wang, F., Huang, L. Y., Sang, T. T., Liu, F., et al. (2015). SIRT1 protects against oxidative stress-induced endothelial progenitor cells apoptosis by inhibiting FOXO3a via FOXO3a ubiquitination and degradation. J. Cell. Physiol. 230, 2098-2107. doi: 10.1002/jcp.24938

Watroba, M., Maslinska, D., and Maslinski, S. (2012). Current overview of functions of FoxO proteins, with special regards to cellular homeostasis, cell response to stress, as well as inflammation and aging. Adv. Med. Sci. 57, 183-195. doi: 10.2478/v10039-012-0039-1

Wei, Q., Shi, F., He, J., Xie, C., Xu, K., Zhang, W., et al. (2012). Effects of exogenous 17beta-estradiol on follicular development in the neonatal and immature mouse in vivo. Reprod. Med. Biol. 11, 135-141. doi: 10.1007/s12522-012-0122-0

Wei, Q., Wu, G., Xing, J., Mao, D., Hutz, R. J., and Shi, F. (2019). Roles of poly (ADP-ribose) polymerase 1 activation and cleavage in induction of multi-oocyte ovarian follicles in the mouse by 3-nitropropionic acid. Reprod. Fertil. Dev. 31, 1017-1032. doi: 10.1071/RD18406

Weigel, D., Jurgens, G., Kuttner, F., Seifert, E., and Jackle, H. (1989). The homeotic gene fork head encodes a nuclear protein and is expressed in the terminal regions of the Drosophila embryo. Cell 57, 645-658. doi: 10.1016/ 0092-8674(89)90133-5

Wu, G., Wei, Q., Yu, D., and Shi, F. (2019). Neonatal genistein exposure disrupts ovarian and uterine development in the mouse by inhibiting cellular proliferation. J. Reprod. Dev. 65, 7-17. doi: 10.1262/jrd.2018-070

Xia, X. Y., Yu, Y. J., Ye, F., Peng, G. Y., Li, Y. J., and Zhou, X. M. (2020). MicroRNA506-3p inhibits proliferation and promotes apoptosis in ovarian cancer cell via targeting SIRT1/AKT/FOXO3a signaling pathway. Neoplasma 67, 344-353. doi: 10.4149/neo_2020_190517N441

Xiang, Y., Xu, J., Li, L., Lin, X., Chen, X., Zhang, X., et al. (2012). Calorie restriction increases primordial follicle reserve in mature female chemotherapy-treated rats. Gene 493, 77-82. doi: 10.1016/j.gene.2011.11.019
Yan, J., Deng, D., Wu, Y., Wu, K., Qu, J., and Li, F. (2020). Catalpol protects rat ovarian granulosa cells against oxidative stress and apoptosis through modulating the PI3K/Akt/mTOR signaling pathway. Biosci. Rep. 40:BSR20194032. doi: 10.1042/BSR20194032

Yang, J. L., Zhang, C. P., Li, L., Huang, L., Ji, S. Y., Lu, C. L., et al. (2010). Testosterone induces redistribution of forkhead box-3a and down-regulation of growth and differentiation factor 9 messenger ribonucleic acid expression at early stage of mouse folliculogenesis. Endocrinology 151, 774-782. doi: 10.1210/ en.2009-0751

Zhang, T., Du, X., Zhao, L., He, M., Lin, L., Guo, C., et al. (2019). SIRT1 facilitates primordial follicle recruitment independent of deacetylase activity through directly modulating Aktl and mTOR transcription. FASEB J. 33, 14703-14716. doi: 10.1096/fj.201900782R

Zhang, X. M., Li, L., Xu, J. J., Wang, N., Liu, W. J., Lin, X. H., et al. (2013). Rapamycin preserves the follicle pool reserve and prolongs the ovarian lifespan of female rats via modulating mTOR activation and sirtuin expression. Gene 523, 82-87. doi: 10.1016/j.gene.2013.03.039

Zhang, Z., Wang, F., and Zhang, Y. (2019). Expression and contribution of NLRP3 inflammasome during the follicular development induced by PMSG. Front. Cell Dev. Biol. 7:256. doi: 10.3389/fcell.2019.00256

Zhou, X. L., Xu, J. J., Ni, Y. H., Chen, X. C., Zhang, H. X., Zhang, X. M., et al. (2014). SIRT1 activator (SRT1720) improves the follicle reserve and prolongs the ovarian lifespan of diet-induced obesity in female mice via activating SIRT1 and suppressing mTOR signaling. J. Ovarian Res. 7:97. doi: 10.1186/s13048014-0097-z

Conflict of Interest: The authors declare that the research was conducted in the absence of any commercial or financial relationships that could be construed as a potential conflict of interest.

Copyright (c) 2020 Zhang, Lin, Zhao and Wang. This is an open-access article distributed under the terms of the Creative Commons Attribution License (CC BY). The use, distribution or reproduction in other forums is permitted, provided the original author(s) and the copyright owner(s) are credited and that the original publication in this journal is cited, in accordance with accepted academic practice. No use, distribution or reproduction is permitted which does not comply with these terms. 